07,12

\title{
Тепловые флуктуации и резонансные свойства сканирующих зондов на основе углеродных нанотрубок
}

\author{
(C) Д.В. Чалин, М.В. Авраменко \\ Южный федеральный университет, \\ Ростов-на-Дону, Россия \\ ฯ E-mail: avramenko.marina@gmail.com \\ (Поступила в Редакцию 19 сентября 2018 г. \\ В окончательной редакции 9 октября 2018 г.)
}

\begin{abstract}
Проектирование кантилеверов и микроэлектромеханических систем с использованием углеродных нанотрубок неразрывно связано с изучением их тепловых флуктуаций и резонансных свойств. В настоящей работе для одностенных углеродных нанотрубок производится расчет тепловых флуктуаций и спектральной плотности интенсивности колебаний в рамках континуальной модели упругих цилиндрических мембран, не обладающих макроскопической толщиной. Найдены налагаемые на геометрию такого зонда условия, выполнение которых обеспечивает как стабильность его работы, так и высокое латеральное разрешение, определяющееся только радиусом нанотрубки и не зависящее от величины ее тепловых колебаний. Определены самые интенсивные резонансные моды, дающие наибольший вклад в тепловые флуктуации углеродных нанотрубок, а также получено аналитическое выражение для их частот.
\end{abstract}

DOI: 10.21883/FTT.2019.03.47244.237

\section{1. Введение}

Представляя собой один из самых мощных и современных методов исследования рельефа поверхностей различных материалов с разрешением вплоть до атомарного, атомно-силовая микроскопия (АСM) [1] является востребованной не только в физике, химии и биологии, но и в различных междисциплинарных областях, таких как материаловедение и биофизика [2,3]. К сожалению, традиционно используемые в АСМ кантилеверы из кремния и нитрида кремния, представляющие собой балку с вытравленным острым наконечником (иглой), имеют ряд ограничений [4]: в частности, они слабо пригодны для исследования материалов со сложной биологической структурой (например, белковых макромолекул) или значительной пористостью. В качестве их идеальной замены уже давно рассматриваются кантилеверы, в которых в роли иглы выступает индивидуальная углеродная нанотрубка (УНТ): они обладают малым диаметром (в среднем, 1-3 nm), высоким аспектным отношением и огромной механической прочностью в сочетании с гибкостью. Поэтому при помощи таких зондов возможно не только повысить чувствительность и разрешающую способность атомно-силовых микроскопов, но и продлить их срок службы в принципе и минимизировать потенциальное разрушающее воздействие зонда на образец [5]. Следует отметить, что перечисленные свойства УНТ также могут послужить основой для создания нового поколения микроэлектромеханических систем (МЭМС), которые все больше и больше находят свое применение в сферах робототехники и искусственного интеллекта [6-8].

Проектирование АСМ-зондов и МЭМС на основе УНТ неразрывно связано с изучением их тепловых флуктуаций и резонансных свойств, а также температурных зависимостей указанных характеристик, поскольку внутренние тепловые колебания УНТ могут достигать по своему абсолютному значению величины порядка радиуса нанотрубки $[9,10]$ и будут неизбежно вызывать в устройствах связанный с ними шум. Исследованию указанной проблемы посвящено несколько экспериментальных работ [9-11], авторы которых проводили наблюдение флуктуаций УНТ и оценку их механических параметров при помощи электронной микроскопии. Теоретических работ по данным вопросам насчитывается более десятка: некоторые из них используют для расчетов молекулярную динамику $[12,13]$ или ее сочетание [14-17] с моделями упругого стержня Эйлера и Тимошенко $[18,19]$ для проверки своих расчетов, другие - модели Эйлера и Тимошенко $[18,19]$ в сочетании [20] с квантовой теорией или континуальные [21,22] и квазиконтинуальные $[23,24]$ модели, учитывающие межатомные потенциалы взаимодействия. Подходы, использующие для расчетов методы молекулярной динамики и межатомные потенциалы взаимодействия в УНТ, в любом случае ограничены большими объемами вычислений и необходимостью одновременного учета целого ряда различных параметров. В свою очередь, в силу того, что амплитуда тепловых флуктуаций УНТ при высоких температурах обратно пропорциональна частоте ее колебаний [14], различные модели, не учитывающие дискретную структуру УНТ (в том числе стержневые), должны давать достаточно простое и близкое к реальным величинам описание тепловых флуктуаций УНТ. Тем не менее, стержневые модели учитывают лишь ограниченное количество степеней свободы трубки: одну степень свободы в модели Эйлера и две - в модели Тимошенко $[18,19]$, что все-таки не позволяет получить 
высокого уровня точности при расчете тепловых флуктуаций. Поэтому в настоящей работе мы предлагаем изучить тепловые флуктуации и резонансные свойства УНТ с позиции такой континуальной теории [25], которая рассматривает УНТ как упругую цилиндрическую мембрану, не имеющую макроскопической толщины, и уже зарекомендовала себя как удобный инструмент для изучения с высокой точностью низкочастотного фононного спектра как индивидуальных, так и находящихся под воздействием окружения одностенных и двустенных УНТ [25], а также их низкотемпературной теплоемкости и баллистической теплопроводности [26-28].

Работа структурирована следующим образом. В разделе 2 мы развиваем модель [25] низкочастотной динамики одностенных УНТ с целью ее последующего (раздел 3) применения для описания их тепловых флуктуаций и резонансных свойств. Работа завершается представлением результатов и их обсуждением (раздел 4) и Заключением.

\section{2. Модель низкочастотной динамики одностенных УНТ}

В рамках развиваемой нами континуальной модели [25] графен и свернутая из него одностенная углеродная нанотрубка (ОУНТ) с самого начала рассматриваются как двумерные мембраны в один атом толщиной. Плотность свободной энергии такой мембраны записывается в виде, общем для локально изотропных двумерных мембран разной формы

$$
g=\frac{\lambda}{2}\left(\varepsilon_{i i}\right)^{2}+\mu \varepsilon_{i j}^{2}+2 K(\Delta H)^{2},
$$

где $\lambda$ и $\mu-$ двумерные аналоги коэффициентов Ламе, $K$ - прогибная упругость (изгибная жесткость), $\Delta H=H-H_{0}, H_{0}-$ равновесная средняя кривизна мембраны, $H$ - кривизна, приобретаемая мембраной в результате ее деформации, $\varepsilon_{i j}$ - двумерный тензор деформации, зависящий в общем случае от $H_{0}$ и трехмерного поля смещений мембраны $\mathbf{u}=\left(u_{r}, u_{\varphi}, u_{z}\right)$. Последнее в цилиндрической системе координат зависит от угла $\varphi$ и переменной $z$, равной расстоянию вдоль оси недеформированной мембраны. Изменение кривизны, линеаризованное по полю смещений и его производным, принимает вид: $\Delta H=-\Delta_{s} u_{r} / 2 R^{2}$, где $\Delta_{s}=1+\partial_{\varphi}^{2}+R^{2} \partial_{z}^{2}$. Двумерный тензор деформации в цилиндрической системе координат записывается как

$$
\varepsilon_{i j}=\left[\begin{array}{cc}
\frac{1}{R}\left(\frac{\partial u_{\varphi}}{\partial \varphi}+u_{r}\right) & \frac{1}{2}\left(\frac{\partial u_{\varphi}}{\partial z}+\frac{1}{R} \frac{\partial u_{z}}{\partial \varphi}\right) \\
\frac{1}{2}\left(\frac{\partial u_{\varphi}}{\partial z}+\frac{1}{R} \frac{\partial u_{z}}{\partial \varphi}\right) & \frac{\partial u_{z}}{\partial z}
\end{array}\right] .
$$

Отметим, что связь (в форме последнего слагаемого (1)) между изменением средней кривизны мембраны и энергией ее изгиба была установлена еще в пионерских работах Канхама [29] и Хелфриха [30] более
40 лет назад. Плотность свободной энергии (1) тождественна аналогичным выражениям из работ [25-28] и, с точностью до определения упругих модулей и выбора способа параметризации поля смещений, эквивалентна [31]. Однако, согласно результатам [32], энергия изгиба цилиндрической мембраны может задаваться и значительно более сложным выражением, куда дополнительно к оператору $\Delta_{s}$ входят еще два дифференциальных инварианта. Тем не менее, решения уравнений, получаемые на основе моделей [25] и [32], полностью совпадают численно.

Разумеется, выражение (1) для плотности свободной энергии двумерной мембраны не является единственно возможным - из дифференциальной геометрии известно как минимум несколько допустимых квадратичных инвариантов, которые можно включить в него. Например, таким инвариантом может быть локальное изменение гауссовой кривизны цилиндрической мембраны [33]. Однако, на наш взгляд, наиболее разумным является включение в выражение (1) лишь одного такого инварианта, представляющего собой произведение изменения средней кривизны мембраны $\Delta H$, относительного изменения площади ее поверхности $\varepsilon_{i i}$ и некоторой константы упругости, которую мы обозначим как $D$. Тогда плотность свободной энергии цилиндрической мембраны может быть переписана в виде

$$
g=\frac{\lambda}{2}\left(\varepsilon_{i i}\right)^{2}+\mu \varepsilon_{i j}^{2}+2 K(\Delta H)^{2}+D \Delta H \varepsilon_{i i}
$$

Последнее слагаемое в выражении (2), описывающее плотность энергии, связанную с локальным изменением плотности цилиндрической мембраны, оказывается запрещенным для плоской мембраны из соображений симметрии: действительно, при отражении в плоскости мембраны данное слагаемое меняло бы свой знак. Также следует отметить, что введение слагаемого $D \Delta H \varepsilon_{i i}$ в выражение (2) при определенном выборе коэффициента $D$ дает возможность исключить вклад энергии изгиба в те деформации мембраны, которые сохраняют ее цилиндрическую форму. Например, полагая постоянную $D$ равной $K / 2 R$, частота радиально-дыхательной моды (RBM) одностенной УНТ уже не зависит от изгибной упругости $K$. К более подробному обсуждению возможных значений коэффициента $D$ мы вернемся в конце настоящего раздела.

Уравнения движения мембраны, решения которых определяют зависимости поля смещений $\mathbf{u}$ от координат и времени, находятся путем вариации функционала

$$
A=\int\left(g(\mathbf{u})-\frac{\rho}{2} \dot{\mathbf{u}}^{2}\right) d S d t,
$$

где $t-$ время, $d S$ - элемент площади поверхности и $\rho$ - поверхностная плотность мембраны. Чтобы получить уравнения движения, мы подставляем $d S=R d z d \varphi$, 
$\Delta H$ и $\varepsilon_{i j}$ в выражение (3) и находим вариацию. Получившиеся уравнения имеют следующий вид

$$
\begin{aligned}
\rho \ddot{u}_{r} R= & -(\lambda+2 \mu)\left(\frac{u_{r}}{R}+\frac{1}{R} \frac{\partial u_{\varphi}}{\partial \varphi}\right)-\lambda \frac{\partial u_{z}}{\partial z}-K \frac{1}{R^{3}} \Delta_{s}^{2} u_{r}-D \frac{1}{R} \Delta_{s}\left(2 \frac{u_{r}}{R}+\frac{1}{R} \frac{\partial u_{\varphi}}{\partial \varphi}+\frac{\partial u_{z}}{\partial z}\right), \\
& \rho \ddot{u}_{\varphi} R=\frac{\lambda+2 \mu}{R}\left(\frac{\partial u_{r}}{\partial \varphi}+\frac{\partial^{2} u_{\varphi}}{\partial \varphi^{2}}\right)+(\lambda+\mu) \frac{\partial^{2} u_{z}}{\partial \varphi \partial z}+\mu R \frac{\partial^{2} u_{\varphi}}{\partial z^{2}}+D \frac{1}{R^{2}} \Delta_{s} \frac{\partial u_{r}}{\partial \varphi}, \\
& \rho \ddot{u}_{z} R=(\lambda+2 \mu) R \frac{\partial^{2} u_{r}}{\partial z^{2}}+(\lambda+\mu) \frac{\partial^{2} u_{\varphi}}{\partial \varphi \partial z}+\lambda \frac{\partial u_{r}}{\partial z}+\frac{\mu}{R} \frac{\partial^{2} u_{z}}{\partial \varphi^{2}}+D \frac{1}{R} \Delta_{s} \frac{\partial u_{r}}{\partial z} .
\end{aligned}
$$

Решения системы (4) мы будем искать в виде плоских волн $\mathbf{u}(\varphi, z)=\mathbf{A} \exp [i(k z+n \varphi-\omega t)]$, где $k-$ одномерный волновой вектор, $n$ - волновое число, имеющее смысл количества осцилляций по периметру УНТ, $\omega-$ круговая частота. Подставляя данное выражение в систему уравнений (4), получаем динамическую матрицу системы $m_{i j}$, имеющую вид

$$
\left[\begin{array}{ccc}
\frac{\lambda+2 \mu}{R}+\frac{K X^{2}}{R^{3}}-\frac{2 D X}{R^{2}}-R \rho \omega^{2} & i\left(\frac{\lambda+2 \mu}{R}-\frac{D X}{R^{2}}\right) n & i\left(\lambda-\frac{D X}{R}\right) k \\
-i\left(\frac{\lambda+2 \mu}{R}-\frac{D X}{R^{2}}\right) n & \frac{(\lambda+2 \mu) n^{2}}{R}+\mu R k^{2}-R \rho \omega^{2} & (\lambda+\mu) n k \\
-i\left(\lambda-\frac{D X}{R}\right) k & (\lambda+\mu) n k & (\lambda+2 \mu) k^{2} R+\frac{\mu n^{2}}{R}-R \rho \omega^{2}
\end{array}\right],
$$

где $X=R^{2} k^{2}+n^{2}-1$. Решения уравнения $\operatorname{Det}\left|m_{i j}\right|=0$ определяют три действительных закона дисперсии в ОУНТ $\omega_{\alpha}=\omega_{\alpha}(k, n), \alpha=1 \ldots 3$.

Чтобы численно найти решения системы (4), удобно воспользоваться экспериментальной системой единиц измерения [25], в которой размеры УНТ задаются в $\mathrm{nm}$, длина волнового вектора $k-$ в $\mathrm{nm}^{-1}$, a решения $\operatorname{Det}\left|m_{i j}\right|=0$ определяют частоты в $\mathrm{cm}^{-1}$. Принимая во внимание $\rho \approx 0.762 \mathrm{mg} \mathrm{m}^{-2}$, также можно получить приведенные материальные константы: $\lambda / \rho \approx 2400 \mathrm{~cm}^{-2} \mathrm{~nm}^{2}, \mu / \rho \approx 5200 \mathrm{~cm}^{-2} \mathrm{~nm}^{2}$, $K / \rho \approx 12.5 \mathrm{~cm}^{-2} \mathrm{~nm}^{4}$ [25]. Отметим, что коэффициент $D$ по своей абсолютной величине должен удовлетворять условиям $\lambda R \gg D, \mu R \gg D$, иначе вклад слагаемого $D \Delta H \varepsilon_{i i}$ в энергию самых низкочастотных изгибных мод станет несоразмерно большим, что подтверждается соответствующими расчетами фононных спектров УНТ. Также в результате расчетов оказывается, что верхняя граница для коэффициента $D$, при которой наша модель еще устойчива, определяется условием $|D|<15 K / R$. Однако наиболее удобно выбрать значение постоянной $D$ таким образом, чтобы частота радиальнодыхательной моды ОУНТ (определяемая равенством $m_{11}=0$ для $n=0$ и $\left.k=0[25,26]\right)$ не зависела от $K$ и $D$, поэтому в дальнейшем мы полагаем, что $D=K / 2 R$.

Очевидно, что любая континуальная модель может быть применима, если длина волны рассматриваемых колебаний существенно больше межатомных расстояний. Так как волновое число $n$ равно количеству осцилляций, которые делает рассматриваемая волна при обходе по периметру трубчатой мембраны, то фононы в ней в зависимости от $n$ и $k$ можно характеризовать длиной эффективного волнового вектора

$$
k_{e f f}=\sqrt{k^{2}+\left(\frac{n}{R}\right)^{2}} .
$$

Длина (6), в свою очередь, определяет эффективную длину волны $2 \pi / k_{e f f}$, которая должна быть в несколько раз больше межатомного расстояния в структуре, что и является главным ограничением континуальной модели. Учтя, что расстояние между атомами в решетке графена составляет $0.142 \mathrm{~nm}$, мы считаем континуальную модель применимой в случае $k_{e f f} \leq k_{\text {lim }}$, где $k_{\text {lim }} \approx 11 \mathrm{~nm}^{-1}$. Тогда максимальные значения волновых чисел $k$ и $n$ определяются как $k_{\max }=k_{l i m} / \sqrt{2} \approx 8 \mathrm{~nm}^{-1}$, $n_{\max }=\operatorname{round}\left(k_{\max } \cdot R\right)$, где функция $\operatorname{round}(x)$ округляет $x$ до целого значения.

\section{3. Методика расчета тепловых флуктуаций и резонансных свойств ОУНТ}

Перейдем к непосредственному расчету тепловых флуктуаций и резонансных свойств ОУНТ. Для этого сначала получим выражение для свободной энергии мембраны в виде квадратичной функции амплитуд поля смешения, которое представим в виде следующего ряда Фурье:

$$
\begin{aligned}
\mathbf{u}_{j}(\varphi, z) & =\frac{1}{\sqrt{2}} \\
& \times \sum_{n, m=-\infty}^{\infty} \sum_{\alpha=1}^{3}\left(A_{\alpha, n, m}^{j} \mathbf{e}_{\alpha}^{j} \exp \left[i\left(k_{m} z+n \varphi\right)\right]+\text { k.c. }\right),
\end{aligned}
$$

где $k_{m}=2 \pi m / L, L$ - длина УНТ, $n$ и $m$ - целые волновые числа, $A_{\alpha, n, m}^{j}, j=r, \varphi, z-$ комплексные амплитуды соответствующих гармоник разложения, $\mathbf{e}_{\alpha}^{j}-$ 
$j$-ая компонента вектора поляризации моды $\alpha$, индекс $\alpha$ принимает значения от 1 до 3 , поскольку в УНТ могут возбуждаться нормальные колебания с тремя различными ортогональными направлениями поляризации. Подставляя формулу (6) в выражение (2) и проводя интегрирование по площади нанотрубки, получаем ее упругую энергию

$$
F=2 \pi R L \sum_{n, m=-\infty}^{\infty} \sum_{\alpha=1}^{3} \sum_{i, j} M_{i j}(n, m) A_{\alpha, n, m}^{i} A_{\alpha, n, m}^{j *}
$$

где $M_{i j}(n, m)$ представляет собой матрицу вида

$$
\left[\begin{array}{ccc}
\frac{\lambda+2 \mu}{R^{2}}+\frac{K X(X-1)}{R^{4}} & i\left(\frac{\lambda+2 \mu}{R^{2}}-\frac{K X}{2 R^{4}}\right) n & \frac{i k}{R}\left(\lambda-\frac{K X}{2 R^{2}}\right) \\
i\left(\frac{\lambda+2 \mu}{R^{2}}-\frac{K X}{2 R^{4}}\right) n & \frac{(\lambda+2 \mu) n^{2}}{R^{2}}+\mu k^{2} & \frac{(\lambda+\mu)}{R} n k \\
-\frac{i k}{R}\left(\lambda-\frac{K X}{2 R^{2}}\right) & \frac{(\lambda+\mu)}{R} n k & (\lambda+2 \mu) k^{2}+\frac{\mu n^{2}}{R^{2}}
\end{array}\right] .
$$

В свою очередь, упругая энергия ОУНТ (7) должна равняться средней энергии возбуждения колебательных уровней ОУНТ, выражающейся с помощью распределения Бозе-Эйнштейна как

$$
E=\sum_{n, m=-\infty}^{\infty} \sum_{\alpha=1}^{3} \frac{\hbar \omega_{n, m}^{\alpha}}{\exp \left(\frac{\hbar \omega_{n, m}^{\alpha}}{k_{B} T}\right)-1}
$$

где $T$ - температура, а $\hbar$ и $k_{B}$ - постоянные Планка и Больцмана соответственно.

Приравняв выражения (8) и (10), можно выразить квадрат амплитуды поля смещений УНТ, включающий как радиальные, так и тангенциальные смещения в нанотрубке. Принимая во внимание тот факт, что латеральное разрешение изображения, получаемого с помощью УНТ-зонда, определяется теми тепловыми флуктуациями, при которых атомы УНТ смещаются в направлении, перпендикулярном оси нанотрубки, мы сконцентрируем свое внимание на колебаниях УНТ именно в радиальном направлении, среднеквадратичную амплитуду которых мы в дальнейшем и будем именовать флуктуациями УНТ. Выразив квадрат амплитуды радиальной компоненты поля смещений с помощью формул (8) и (10), мы получаем

$$
\left|A_{\alpha, n, m}^{r}\right|^{2}=\frac{\hbar \omega_{n, m}^{\alpha}}{2 \pi R L\left[\exp \left(\frac{\hbar \omega_{n, m}^{\alpha}}{k_{B} T}\right)-1\right] M_{n, m}^{\alpha}}
$$

где величина

$$
\begin{aligned}
M_{n, m}^{\alpha}= & M_{11}+\left|a_{n, m}^{\alpha}\right|^{2} M_{22}+\left|b_{n, m}^{\alpha}\right|^{2} M_{33}+\left(a_{n, m}^{\alpha}-a_{n, m}^{\alpha *}\right) M_{12} \\
& +\left(b_{n, m}^{\alpha}-b_{n, m}^{\alpha *}\right) M_{13}+\left(a_{n, m}^{\alpha *} b_{n, m}^{\alpha}+a_{n, m}^{\alpha} b_{n, m}^{\alpha *}\right) M_{23},
\end{aligned}
$$

а коэффициенты $a_{n, m}^{\alpha}$ и $b_{n, m}^{\alpha}$ показывают отношение тангенциальных компонент амплитуды к радиальной компоненте соответствующей гармоники

$$
a_{n, m}^{\alpha}=\frac{A_{\alpha, n, m}^{\varphi}}{A_{\alpha, n, m}^{r}}, \quad b_{n, m}^{\alpha}=\frac{A_{\alpha, n, m}^{z}}{A_{\alpha, n, m}^{r}} .
$$

Далее мы можем переписать выражение для радиальной компоненты поля смещения в виде

$$
\begin{aligned}
& u_{r}(\varphi, z)=\frac{1}{\sqrt{2}} \\
& \times \sum_{n, m=-\infty}^{\infty} \sum_{\alpha=1}^{3}\left(\left|A_{\alpha, n, m}^{r}\right| \exp \left[i\left(k_{m} z+n \varphi+\beta_{n, m}^{\alpha}\right)\right]+k . c .\right) \\
& =\sqrt{2} \sum_{n, m=-\infty}^{\infty} \sum_{\alpha=1}^{3}\left|A_{\alpha, n, m}^{r}\right| \cos \left(k_{m} z+n \varphi+\beta_{n, m}^{\alpha}\right),
\end{aligned}
$$

где $\beta_{n, m}^{\alpha}=\operatorname{arctg}\left[\left(A_{\alpha, n, m}^{r}-A_{\alpha, n, m}^{r *}\right) /\left(A_{\alpha, n, m}^{r}+A_{\alpha, n, m}^{r *}\right)\right]$ представляет собой фазу для моды $\alpha$ с волновыми числами $m$ и $n$. После возведения данного выражения в квадрат и усреднения по пространственным координатам, мы получаем конечную формулу, определяющую среднеквадратичную амплитуду флуктуаций УНТ:

$$
\left\langle u_{r}\right\rangle=\sqrt{\sum_{n, m=-\infty}^{\infty} \sum_{\alpha=1}^{3}\left|A_{\alpha, n, m}^{r}\right|^{2}}
$$

На основе формул (11) и (12) можно сделать вывод о том, что самые низкочастотные колебания дают наибольший вклад во флуктуации УНТ даже при высоких температурах: поскольку $M_{n, m}^{\alpha} \sim \omega_{\alpha}^{2}(n, m)$, то величина (11) в температурной области $T \gg \hbar \omega / k_{B}$ оказывается обратно пропорциональна квадрату частоты соответствующей моды.

Также следует обсудить роль различных граничных условий при расчете тепловых флуктуаций УНТ. Для простоты рассуждений рассмотрим одномерный случай. Представим струну (или стержень с одной колебательной степенью свободы), движение которой описывается законом $\sim \sin (k x)$. Тогда среднеквадратичная амплитуда колебаний струны с закрепленными концами будет такой же, как в случае струны вдвое большей длины с периодическими граничными условиями. Аналогичным образом, если один конец струны закреплен, а второй свободен, то оказывается, что такая струна будет колебаться точно также, как и струна вдвое большей длины с двумя закрепленными концами. Все вышесказанное легко обобщается на трехмерный случай, когда в системе могут возбуждаться плоские волны с тремя различными направлениями вектора поляризации. Очевидно, что для расчета флуктуаций УНТ в реальном кантилевере нужно пользоваться граничными условиями, при которых один конец УНТ закреплен, а второй свободен, однако, как напрямую следует из вышеописанного примера, для удобства расчетов нами используются периодические граничные условия, что фактически и было учтено в формуле (7).

В заключение настоящего раздела отметим, что не менее важными для АСМ-зонда на основе УНТ являются его резонансные характеристики, поскольку именно они определяют стабильность его работы при измерениях 
посредством колебательных методик [14,15]. Сами же резонансные свойства рассматриваемого зонда напрямую определяются колебательным спектром самой нанотрубки ввиду линейности упругих свойств рассматриваемой системы. Наиболее полную и важную информацию о спектральных характеристиках нанотрубки можно узнать, рассчитав ее спектральную плотностьинтенсивности колебаний $I(\omega)$. Определим данную величину как интенсивность моды с частотой $\omega$ в частотном интервале $d \omega$, т. е. положим

$$
\left\langle u_{r}^{2}\right\rangle=\int I(\omega) d \omega
$$

Пользуясь определением (13), можно численно найти функцию $I(\omega)$ : спектральную плотность колебаний $I\left(\omega_{0}\right)$ в точке $\omega_{0}$ можно рассчитать как среднюю интенсивность колебаний с частотами, лежащими в диапазоне $\left[\omega_{0}-\Delta \omega_{0} / 2 ; \omega_{0}+\Delta \omega_{0} / 2\right]$.

\section{4. Результаты и обсуждение}

Прежде чем перейти непосредственно к результатам наших вычислений тепловых флуктуаций УНТ, необходимо обсудить выбор геометрических параметров нанотрубок для данных расчетов. Поскольку при сканировании зонд взаимодействует с поверхностью образца, длина и радиус нанотрубки должны находиться в определенном соотношении, для того чтобы при контакте с исследуемой поверхностью нанотрубка не теряла свою устойчивость. Воспользовавшись простейшей моделью упругого стержня [34], можно легко определить критическую силу взаимодействия нанотрубки с поверхностью

$$
F_{c r i t}=\frac{\pi^{2} E J}{L^{2}}
$$

где $E$ представляет собой модуль Юнга нанотрубки, $J=\pi R^{4} / 4$ соответствует моменту инерции ее сечения, а $L$ и $R$ обозначают ее длину и радиус, соответственно. Обычно при реальных измерениях данная сила имеет порядок $10^{-8} \mathrm{~N}$ [35]. Тогда, пользуясь условием $F_{\text {crit }}<10^{-8} \mathrm{~N}$ и зная, что модуль Юнга нанотрубки $E=1 \mathrm{TPa}[20]$, находим, что длина нанотрубки должна удовлетворять условию $L<28 R^{2}$ ( $L$ и $R$ в данном выражении измеряются в $\mathrm{nm})$. Например, для ОУНТ $(10,10)$ ее критическая длина $L_{\text {crit }}$ равняется $12.94 \mathrm{~nm}$. Однако это не единственное условие, согласно которому мы должны ограничивать длину УНТ. Поскольку латеральное разрешение изображения определяется непосредственно лишь самим радиусом УНТ-зонда, необходимо, чтобы амплитуда тепловых колебаний $A$ была на порядок меньше радиуса нанотрубки. Комбинируя указанную выше модель упругого стержня с теоремой о равнораспределении энергии по степеням свободы, нетрудно получить следующее выражение, позволяющее оценить

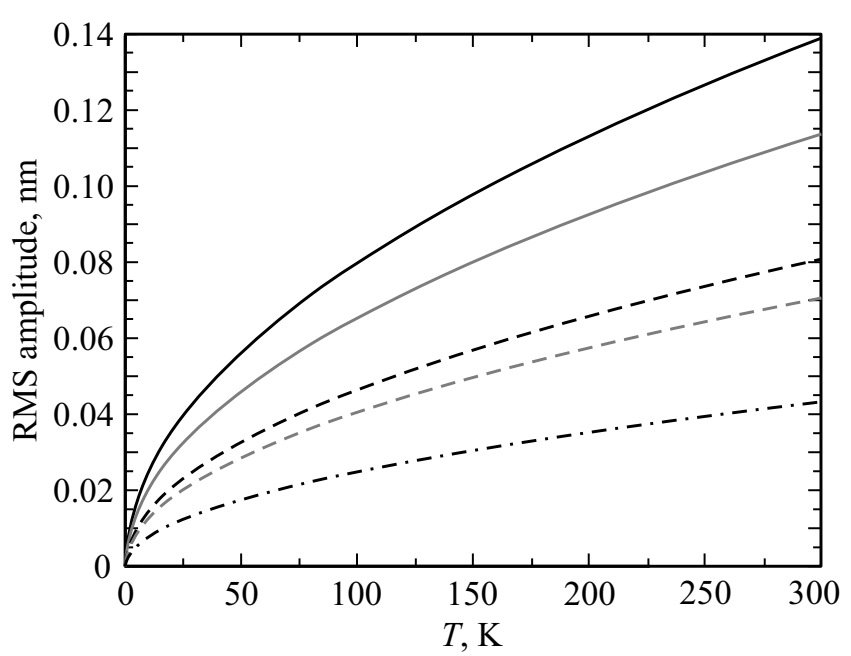

Рис. 1. Зависимость среднеквадратичной амплитуды тепловых флуктуаций от температуры. Сравнение результатов, полученных в рамках нашей модели и модели упругого стержня Эйлера [20]. Кривые, показанные сплошной, штриховой и штрихпунктирной линиями, демонстрируют результаты расчетов для ОУНТ $(26,18),(21,9)$ и $(10,10)$ соответственно. Результаты расчетов в рамках нашей континуальной модели показаны черным, в рамках модели Эйлера - серым.

амплитуду $A$ как

$$
A=\left(\frac{4 k_{B} T L^{3}}{\pi^{4} E J}\right)^{1 / 2} .
$$

Из условия $A<0.1 R$ при $T=300 \mathrm{~K}$ находим, что длина нанотрубки должна удовлетворять условию $L<36 R^{2}$. Таким образом, мы получили результат, согласно которому устойчивый к продольным нагрузкам УНТ-зонд также будет давать достаточно высокое латеральное разрешение изображения, которое главным образом определяется радиусом нанотрубки, а не величиной ее тепловых колебаний.

Определив все особенности выбора геометрии нанотрубки для ее применения в качестве зонда, мы переходим к нашим вычислениям, позволяющим определить величину тепловых флуктуаций УНТ с большей точностью, нежели это удается с помощью выражения (15). На рис. 1 представлена зависимость среднеквадратичной амплитуды тепловых флуктуаций нанотрубки от окружающей температуры. Обычно радиусы синтезируемых ОУНТ лежат в пределах от $0.5 \mathrm{~nm}$ до $1.5 \mathrm{~nm}$, поэтому для расчетов были взяты ОУНТ $(10,10),(21,9)$ и $(26,18)$, их радиусы равны $0.68,1.04$ и $1.5 \mathrm{~nm}$ соответственно. Длины нанотрубок выбирались согласно критерию, полученному из формулы (14), а именно таким образом, чтобы длина каждой УНТ составляла 90\% от ее критической длины, при которой возможна потеря устойчивости нанотрубки при взаимодействии со сканируемой поверхностью. При этом также учтена зависимость флуктуаций от вида граничных условий, 
которая обсуждалась в предыдущем разделе: полученные результаты соответствуют тепловым колебаниям нанотрубок с одним закрепленным концом, а вторым свободным. Таким образом, фактические длины ОУНТ $(10,10),(21,9)$ и $(26,18)$, использованные при расчетах, составляют $46.60,109.02$ и $226.80 \mathrm{~nm}$ соответственно. Во всех случаях при $T=300 \mathrm{~K}$ среднеквадратичная амплитуда флуктуаций $\left\langle u_{r}\right\rangle$ не превышает даже 0.07 части радиуса $R$ УНТ, как и должно быть, согласно предложенным выше критериям выбора геометрических размеров нанотрубки. Для того чтобы амплитуда тепловых колебаний превысила диаметр УНТ, необходимо, чтобы длина нанотрубки была порядка нескольких сотен нанометров или даже микронов. Например, согласно нашим расчетам, флуктуации ОУНТ $(10,10)$, один конец которой закреплен, а второй свободен, достигают величины ее диаметра лишь при длине нанотрубки равной $150 \mathrm{~nm}$.

Далее мы переходим к сравнению нашей континуальной модели упругих мембран в один атом толщиной с моделью упругого стержня Эйлера. Как видно из рис. 1, разница между результатами, полученными в рамках обоих подходов, растет с увеличением длины и радиуса УНТ. Данное явление можно объяснить тем фактом, что с помощью нашей модели можно рассчитать намного большее число низкочастотных мод, принадлежащих различным дисперсионным кривым фононного спектра УНТ, которые также будут давать некоторый вклад в тепловые флуктуации нанотрубки. А именно, наша теория позволяет рассчитать $3 \cdot\left(n_{\max }+1\right)$ дисперсионных веток, где $n_{\max }$ определяется пределами применимости континуального приближения, которые обсуждались в конце раздела 2. При этом, если учесть вырождение по волновому вектору $k$ и волновому числу $n$, получаем, что полное число дисперсионных кривых равно $6 \cdot\left(2 n_{\max }+1\right)$. Более того, так как, согласно формуле (6), $n_{\max }=\operatorname{round}\left(k_{\max } \cdot R\right)$, то с увеличением размеров трубки также увеличивается количество рассчитываемых дисперсионных кривых, и, соответственно, число мод. В свою очередь, в колебательном спектре эйлерова стержня присутствует лишь одна дисперсионная кривая (ее вид будет приведен ниже), в результате чего данная модель позволяет учесть лишь ограниченное число наиболее интенсивных мод, дающих вклад в тепловые флуктуации УНТ.

Далее мы переходим к обсуждению резонансных и спектральных свойств УНТ. На рис. 2 представлена спектральная плотность интенсивности колебаний, рассчитанная для ОУНТ $(10,10)$ длиной $46.6 \mathrm{~nm}$. Спектральная интенсивность $I(\omega)$ в каждой точке $\omega$ определялась как средняя интенсивность мод, попавших в окрестность точки $\Delta \omega$. Общее число мод, рассчитываемых для данной ОУНТ, равняется 3366. Поскольку в низкочастотной области $\left(\omega<50 \mathrm{~cm}^{-1}\right)$ плотность колебаний относительно мала, величина $\Delta \omega$ в наших расчетах полагается равной $10 \mathrm{~cm}^{-1}$, для того чтобы в окрестности любой точки оказалось как минимум две моды.

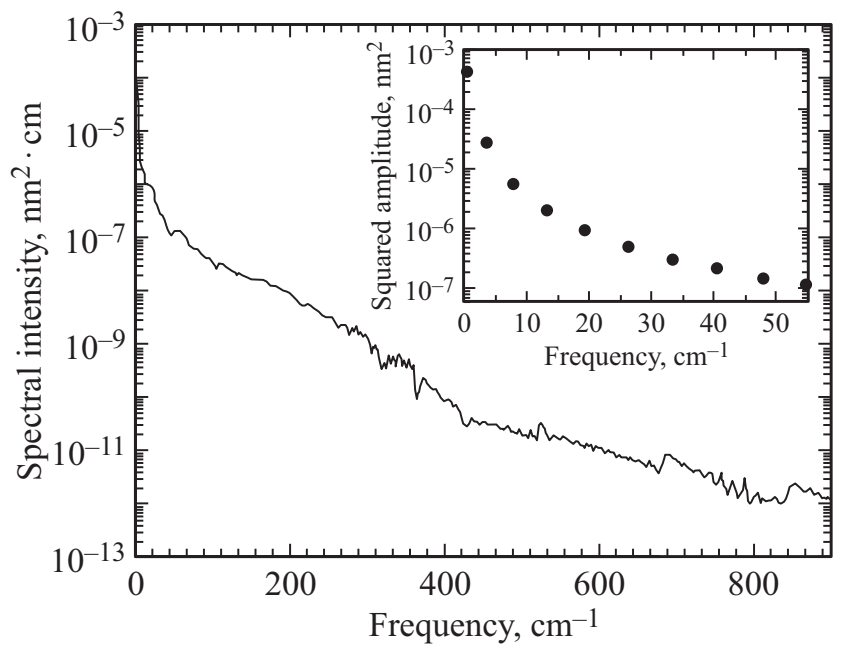

Рис. 2. Спектральная плотность колебаний, рассчитанная для ОУНТ $(10,10)$ длиной $46.6 \mathrm{~nm}$ при температуре $300 \mathrm{~K}$. На вставке показаны интенсивности мод, дающих наибольший вклад в тепловые флуктуации ОУНТ.

Как можно видеть на рис. 2, наибольший вклад в интенсивность флуктуаций сосредоточен в области до $200 \mathrm{~cm}^{-1}$, что относительно близко к частоте радиальной дыхательной моды (равной $166 \mathrm{~cm}^{-1}$ для данной ОУНТ). Именно в этом частотном диапазоне расположена большая часть мод с чисто радиальной поляризацией.

На вставке рис. 2 показаны моды, дающие наибольший вклад в тепловые флуктуации УНТ. В терминах волновых чисел это колебания, принадлежащие дисперсионной кривой в фононном спектре УНТ с $n=1$, $m=1,2,3 \ldots$ Сам закон дисперсии в длинноволновом пределе имеет очень простой вид и записывается как

$$
\omega_{m}=\frac{\omega_{R B M}}{\sqrt{2}} R^{2} k_{m}^{2},
$$

где $\omega_{R B M}=\sqrt{(\lambda+2 \mu) / \rho R^{2}}$ — частота радиальнодыхательной моды, $k_{m}=\alpha m / L-$ одномерный волновой вектор, $\alpha-$ коэффициент, зависящий от вида граничных условий. Для выбранной ОУНТ $(10,10)$ интенсивность данного набора мод составляет $90 \%$ от суммарной интенсивности всех колебаний. С увеличением длины УНТ и, соответственно, уменьшением степени дискретизации волнового вектора, будет возбуждаться все большее число мод, принадлежащих дисперсионной кривой (16). Поэтому при некоторой длине, превышающей критическую $L_{c r i t}$, их вклад в тепловые колебания нанотрубки будет достигать $99 \%$ даже несмотря на то, что в фононном спектре УНТ присутствуют две ветви с линейным законом дисперсии [25]. Их вклад во флуктуации оказывается на несколько порядков меньше, чем у мод (16), ввиду продольной и тангенциальной поляризации нормальных колебаний, принадлежащих данным дисперсионным кривым.

В заключение раздела следует отметить, что модель Эйлера, использовавшаяся в работах [14-24], позволяет 
рассчитать частоты и амплитуды только поперечных колебаний стержня, при этом закон дисперсии, отвечающий данным модам, повторяет закон (16). Однако при выборе определенных геометрических размеров нанотрубки обе рассматриваемые модели могут давать близкие результаты, поскольку вклад мод (16) во флуктуации УНТ может достигать 99\% при длине УНТ, превышающей критическую.

\section{5. Заключение}

В работе проведено исследование тепловых флуктуаций АСМ-зондов на основе углеродных нанотрубок, а также рассчитаны их спектральные характеристики. Для этого была развита континуальная модель, рассматривающая одностенную углеродную нанотрубку как цилиндрическую мембрану в один атом толщиной и учитывающая локальное изменение плотности такой мембраны. Предлагаемый подход позволяет легко рассчитать низкочастотную область фононного спектра нанотрубки, которая оказывается наиболее важной при расчете тепловых флуктуаций УНТ, поскольку их амплитуда обратно пропорциональна частоте колебаний.

Были выявлены условия, определяющие выбор геометрических параметров нанотрубки, выполнение которых обеспечивает стабильность работы зонда и высокое латеральное разрешение, не зависящее непосредственно от величины тепловых колебаний нанотрубки. Были рассчитаны тепловые флуктуации и их температурная зависимость для УНТ различного диаметра, произведен анализ и сравнение выполненных расчетов с вычислениями, полученными с помощью модели упругого стержня Эйлера. Показано, что при выполнении найденных условий, накладываемых на геометрию УНТ-зонда, амплитуда тепловых флуктуации не будет превышать и 0.1 части радиуса нанотрубки. Далее была рассчитана спектральная плотность интенсивности колебаний. В заключение были определены самые интенсивные моды, дающие наибольший вклад в суммарную амплитуду тепловых колебаний, а также найдено аналитическое выражение, определяющее их частоты.

\section{Список литературы}

[1] G. Binnig, C.F. Quate, Ch. Gerber. Phys. Rev. Lett. 56, 9, 930 (1986).

[2] Z. Deng, V. Lulevich, F. Liu, G. Liu. J. Phys. Chem. B 114, $5971(2010)$.

[3] U. Mavera, T. Velnarb, M. Gaberščekd, O. Planinšeke, M. Finšgar. Trac-Trend. An. Chem. 80, 96 (2016).

[4] N.R. Wilson, J.V. Macpherson. Nature Nanotechnol. 4, 483 (2009).

[5] H. Dai, J.H. Hafner, A.G. Rinzler, D.T. Colbert, R.E. Smalley. Nature 384, 147 (1996).

[6] E. Gaura, R. Newman. Smart MEMS and sensor systems. Imperial College Press, London, UK (2006). 539 p.
[7] H.B. Muhammad, C.M. Oddo, L. Beccai, C. Recchiuto, C.J. Anthony, M.J. Adams, M.C. Carrozza, D.W.L. Hukins, M.C.L. Ward. Sensor. Actuat. A-Phys. 165, 221 (2011).

[8] X. Zang, Q. Zhou, J. Chang, Y. Liu, L. Lin. Microelectron. Eng. 132, 192 (2015).

[9] M.M.J. Treacy, T.W. Ebbesen, J.M. Gibson. Nature 381, 678 (1996).

[10] I. Tsioutsios, A. Tavernarakis, J. Osmond, P. Verlot, A. Bachtold. Nano Lett. 17, 3, 1748 (2017).

[11] A. Krishnan, E. Dujardin, T.W. Ebbesen, P.N. Yianilos, M.M.J. Treacy. Phys. Rev. B 58, 14013 (1998).

[12] Z.P. Xu, Q.-S. Zheng, G.H. Chen. Phys. Rev. B 74, 195445 (2006).

[13] A.W. Barnard, V. Sazonova, A.M. van der Zande, P.L. McEuen. P. Natl. Acad. Sci. USA 109, 47, 19093 (2012).

[14] E.H. Feng, R.E. Jones. Phys. Rev. B 81, 125436 (2010).

[15] E.H. Feng, R.E. Jones. Phys. Rev. B 83, 125412 (2011).

[16] L. Wang, H. Hu, W. Guo. Proc. R. Soc. A 466, 2325 (2010).

[17] L.F. Wang, H. Y. Hu. Acta Mech. 223, 2107 (2012).

18] S.P. Timoshenko. Phil. Mag. 41, 744 (1921).

[19] S.P. Timoshenko. History of strength of materials. McGrawHill, N.Y., USA (1953). 452 p.

[20] L. Wang, H. Hu. Proc. R. Soc. A 470, 20140087 (2014).

[21] H. Jiang, B. Liu, Y. Huang, K. C. Hwang. J. Eng. Mater. Technol. 126, 265 (2004).

[22] H. Jiang, Y. Huang, K.C. Hwang. J. Eng. Mater. Technol. 127, 408 (2005).

[23] X. Guo, J.B. Liao, X.Y. Wang. Comput. Mater. Sci. 51, 445 (2012).

[24] X.Y. Wang, X. Guo. Comput. Mater. Sci. 55, 273 (2012).

[25] S.B. Rochal, V.L. Lorman, Yu. I. Yuzyuk. Phys. Rev. B 88, 235435 (2013)

[26] M.V. Avramenko, I.Yu. Golushko, A.E. Myasnikova, S.B. Rochal. Phys. E 68, 133 (2015).

[27] М.В. Авраменко, С.Б. Рошаль. ФТТ 58, 5, 979 (2016).

[28] D.V. Chalin, M.V. Avramenko, S.B. Rochal. Phys. Rev. B 96, 155413 (2017).

[29] P.B. Canham. J. Theor. Biology 26, 1, 61 (1970).

[30] W. Helfrich. Naturforsch. C 98, 11, 693 (1973).

[31] H. Suzuura, T. Ando. Phys. Rev. B 65, 235412 (2002).

[32] S.V. Goupalov. Phys. Rev. B 71, 085420 (2005).

[33] L.D. Landau, E.M. Lifshitz. Theory of Elasticity. Pergamon Press, Oxford, UK (1989). 188 p.

[34] Н.А. Алфутов. Основы расчета на устойчивость упругих систем. Машиностроение, М. (1978). 312 с.

[35] Handbook of Micro/Nanotribology / Ed. Bhushan Bharat. 2 nd. ed, Boca Raton etc, CRC press. (1999). 859 c.

Редактор Т.Н. Василевская 